\title{
Computer Based Bone Breakage Detection using Machine Learning Techniques
}

\author{
P. Neelakanteswara, G.Kalyan Chakravarthy, Ram Kumar Madupu, Dorababu Sudarsa
}

\begin{abstract}
X$-Ray is one of the most commonly used medium to extract the images of any bone in the body.Fracture of a bone is most common in recent days due to accidents or any means.In order to detect whether there is a fracture or not the orthopaedics suggest for $x$-ray.In many places due to more patients there might be a delay of doctor consult which may leads to the increase in the severity of problem.In order to avoid this we have proposed an automatic bone fracture detection system where a system is trained about the fractures and further used to detect the fractures in a bone in the $x$-ray images.ANN,PNN.BPNN are the classifiers used for bone fracture detection where BPNN has given more prominent results compared to ANN and PNN with an accuracy of $82 \%$.
\end{abstract}

Keywords : ANN,PNN,BPNN,X-Ray,Fracture,

\section{INTRODUCTION}

Computer supported illness recognition assume a crucial job in a wide scope of utilizations and administrations in everyday exercises. The most significant quality objective of such a framework is speed and high precision in ailment location. This is progressively significant in radiology division, where hundreds and several pictures are to be analyzed for different diseases including bone break, contamination and area of remote articles. Out of this, bone crack identification is the most much of the time utilized task, on the grounds that the populace influenced by this affliction has expanded because of the expanded reports of mishaps and because of osteoporosis. As per the market report of [ IS], the crack highlights are more in Indian clinic records and the rate has expanded 3-overlap in the course of recent decades with more than 4.4 laTh individuals and is relied upon to increment to in excess of 6 laTh in 2020 [21]. Among cracks, programmed discovery in tibia is viewed as more chaUenging in light of the fact that they are extraordinary and variable in introduction and their results are flighty. Tibia

Revised Manuscript Received on December 30, 2019.

* Correspondence Author

P.Neelakanteswara*, Department of Computer Science and Engineering, Koneru Lakshmaiah Education Foundation, Vaddeswaram, AP, India. Email:neelakanta2601@gmail.com

G Kalyan Chakravarthy, Department of Computer Science and Engineering, Koneru Lakshmaiah Education Foundation, Vaddeswaram, AP, India. Email:send2chakri3@gmail.com

Ram Kumr Madupu, Department of Computer Science and Engineering, Koneru Lakshmaiah Education Foundation, Vaddeswaram, AP, India.. Email:ramramkumar.one@gmail.com

Dorababu Sudarsa, Department of Computer Science and Engineering, Koneru Lakshmaiah Education Foundation, Vaddeswaram, AP, India. Email: dorababu.sudarsa@gmail.com

(c) The Authors. Published by Blue Eyes Intelligence Engineering and Sciences Publication (BEIESP). This is an open access article under the CC BY-NC-ND license (http://creativecommons.org/licenses/by-nc-nd/4.0/) breaks are the most widely recognized long bone crack bookkeeping to more than 20 percent inhabitance of medical clinic wards [9]. As indicated , by and large 26 tibia cracks happen per 1,00,000 populaces for every year.The various rates require the human services experts to dissect gigantic number of x-beam pictures. As a result of consistently examining pictures, instances of miss conclusion happen. A miss is defmed as either inability to see a noteworthy fmding, or joining the erroneous criticalness to a fmding that is promptly observed. A high miss-conclusion rate will bring about low quality. in medicinal services and time-postponed treatment and must be maintained a strategic distance from. Every one of these realities have inspired us to concentrate on the proposed break recognizable proof and recognition framework that can consequently decide the nearness and nonattendance of cracks and if present, techniques that could find the breaks in a bone x-beam picture. Arrangement, a much of the time utilized information mining strategy, has been utilized generally to identify the nearness of crack for as far back as not many decades .These frameworks join different highlights (like shape, surface and shading) separated from x-beam pictures and AI calculations to distinguish issues.

As per [31] when an ideal arrangement of highlights that can portray the picture information is given, the exactness of the resultant characterization this we have proposed an automatic bone fracture detection system where a system is trained about the fractures and further used to detect the fractures in a bone in the $\mathrm{x}$-ray images.ANN,PNN.BPNN are the classifiers used for bone fracture detection where BPNN has given more prominent results compared to ANN and PNN with an accuracy of $82 \%$.relies upon the classifier embraced. A few arrangements utilizing numerous classifiers and afterward the different outcomes can be combined to acquire an ideal order. Utilizing numerous classifiers (either various kinds of classifiers or various launches of a similar classifier) improve the achievement pace of a characterization model. This procedure is named as 'outfit arrangement' or 'combination order' or 'numerous grouping framework'. As indicated by [31], instinctively, combination characterization permits the various needs of a troublesome issue to be dealt with by classifiers fit to those specific needs. Scientifically, combination classifier give an additional level of opportunity in the traditional predisposition/difference tradeoff, permitting arrangements that would be troublesome (if not difficult) to reach with just a solitary classifier.

A general outfit grouping framework when applied to classifY $\mathrm{x}$-beam pictures is appeared .

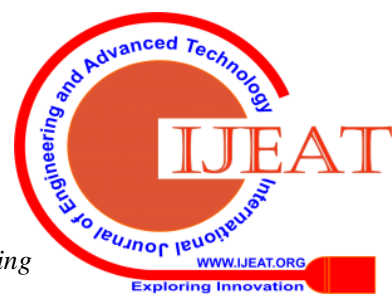




\section{Computer Based Bone Breakage Detection using Machine Learning Techniques}

The precision of such a troupe X-Ray is one of the most commonly used medium to extract the images of any bone in the body.Fracture of a bone is most common in recent days due to accidents or any means. In order to detect whether there is a fracture or not the orthopaedics suggest for $\mathrm{x}$-ray.In many places due to more patients there might be a delay of doctor consult which may leads to the increase in the severity of problem.In order to avoid this we have proposed an automatic bone fracture detection system where a system is trained about the fractures and further used to detect the fractures in a bone in the $x$-ray images.ANN,PNN.BPNN are the classifiers used for bone fracture detection where BPNN has given more prominent results compared to ANN and PNN with an accuracy of $82 \%$.model relies upon a few elements like (I) Number and sort of classifiers (ii) Features utilized (iii) Partitioning strategy (Training and Testing sets) (iv) The conglomeration technique and (v) Type of preparing. An effective ensembling relies upon the structure of the individual classifiers, determination of classifier and plan of the combination system. Concentrates detailed have

\section{EXISTING METHOD}

This segment presents surveys of the different strategies that exist in the region of numerous order problem.A related strategy is the Random Forest calculation by [3], which has exhibited high characterization execution in numerous fields of research [1]. A Random Forest consolidates Bagging and a particular type of RSM where arbitrary include subset determination is performed at every hub of a part choice tree. Revolution Forest [34], a various classifier dependent on turns of the element space through Principal Component Analysis (PCA). The reason for Rotation Forest is to expand the individual classifier execution and the decent variety inside the different arrangement process. Assorted variety is accomplished for every classifier by applying highlight extraction, while one attempts to build the exhibition by utilizing all foremost parts and preparing the model overall information set.A second procedure to expand grouping execution is to choose an elective base classifier calculation. Numerous studies have proposed combination dependent on elective base classifiers, for example, Artificial Neural Networks [40], Support Vector Machines [22], parametric relapse procedures [33] and nonparametric relapse systems [2]. Computer supported illness recognition assume a crucial job in a wide scope of utilizations and administrations in everyday exercises. The most significant quality objective of such a framework is speed and high precision in ailment location. This is progressively significant in radiology division, where hundreds and several pictures are to be analyzed for different diseases including bone break, contamination and area of remote articles. Out of this, bone crack identification is the most much of the time utilized task, on the grounds that the populace influenced by this affliction has expanded because of the expanded reports of mishaps and because of osteoporosis. As per the market report of [ IS], the crack highlights are more in Indian clinic records and the rate has expanded 3-overlap in the course of recent decades with more than 4.4 laTh individuals and is relied upon to increment to in excess of 6 laTh in 2020 [21].

\section{PROPOSED METHOD}

This consists of totally six steps

1)Input

2)Second is Preprocessing

3)Second is Segmentation

4)Fourth is Feature extraction

5)Fifth is training the neural network

6)Detection

$\mathrm{X}$-Ray is one of the most commonly used medium to extract the images of any bone in the body.Fracture of a bone is most common in recent days due to accidents or any means.In order to detect whether there is a fracture or not the orthopaedics suggest for $\mathrm{x}$-ray.In many places due to more patients there might be a delay of doctor consult which may leads to the increase in the severity of problem.In order to avoid this we have proposed an automatic bone fracture detection system where a system is trained about the fractures and further used to detect the fractures in a bone in the $\mathrm{x}$-ray images.ANN,PNN.BPNN are the classifiers used for bone fracture detection where BPNN has given more prominent results compared to ANN and PNN with an accuracy of $82 \%$.are utilized to produce the element vector. The surface highlights gathered are GLCM (Gray Level CoOccurrence Matrix) highlights, to be specific, Contrast, Homogeneity, Energy, Entropy, Mean, Variance, Standard Deviation, Correlation, Gabor direction (GO), Markov Random Field (MRF), and force slope course (IGD). The shape highlights are extricated utilizing a Fast Hough Transformation proposed by [16]. The highlights gathered are organized in a two-dimensional grid where every section speaks to an element extricated and the column speaks to different highlights that speak to the fragmented x-beam picture. The last segment is treated as an objective mark section, having a Boolean estimation of 0 to demonstrate the nonattendance of crack and 1 to show the nearness of break. Utilizing these 12 highlights, three list of capabilities vectors are made. The frrst one comprises of just surface highlights, second have just shape highlights and the third set have a shape and surface highlights and are alluded to as FS I, FS2 and FS I2 separately in this paper. Computer supported illness recognition assume a crucial job in a wide scope of utilizations and administrations in everyday exercises. The most significant quality objective of such a framework is speed and high precision in ailment location. This is progressively significant in radiology division, where hundreds and several pictures are to be analyzed for different diseases including bone break, contamination and area of remote articles. Out of this, bone crack identification is the most much of the time utilized task, on the grounds that the populace influenced by this affliction has expanded because of the expanded reports of mishaps and because of osteoporosis. As per the market report of [ IS], the crack highlights are more in Indian clinic records and the rate has expanded 3-overlap in the course of recent decades with more than 4.4 laTh individuals and is relied upon to increment to in excess of 6 laTh in 2020 [21].

Published By:

Blue Eyes Intelligence Engineering

DOI: 10.35940/ijeat.B3827.129219

Journal Website: www.ijeat.org 
The present investigation considers three classifiers in particular, Support Vector Machine (SVM) [15], K-Nearest Neighbor (KNN) 10] and Back Propagation Artificial Neural Network (BPNN) [7]. Every one of the classifiers are structured as paired classifiers, where the order result is either 'Crack .

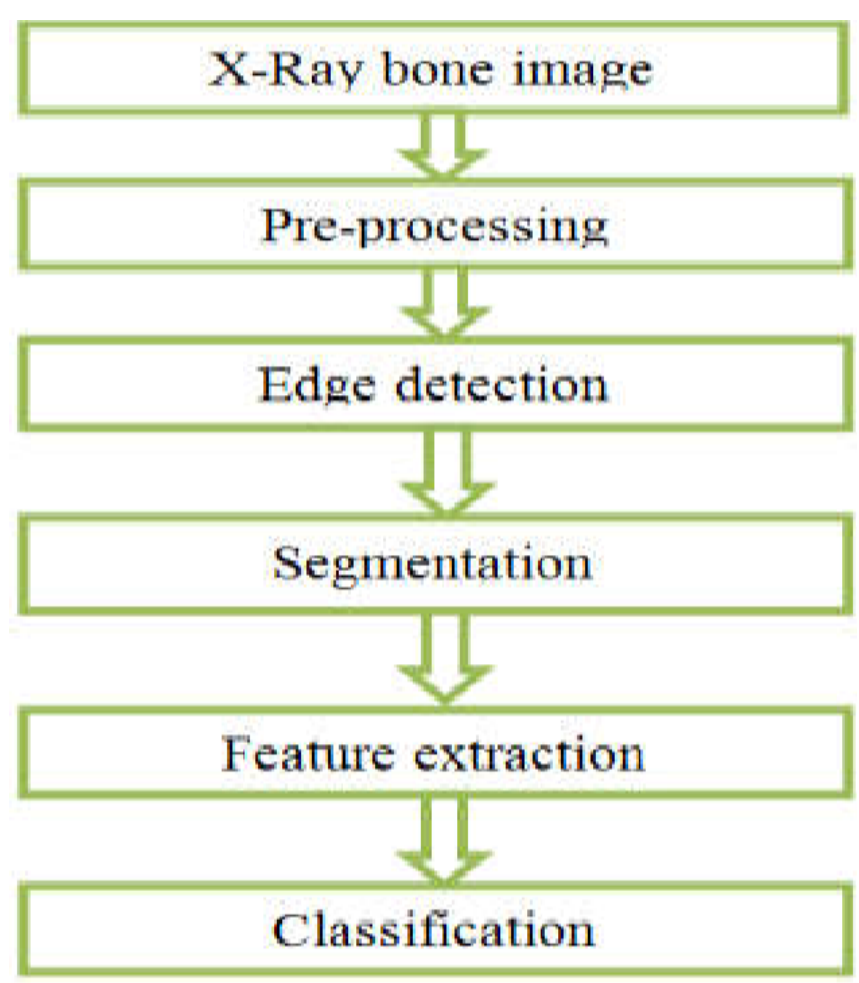

PROPOSED SYSTEM

\section{RESULTS:}

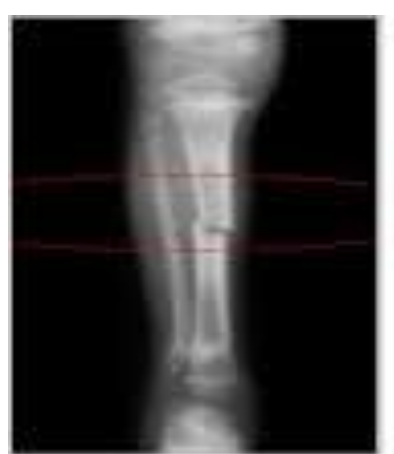

(a)

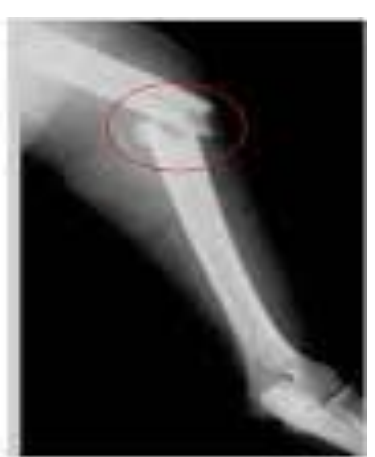

\{b\}

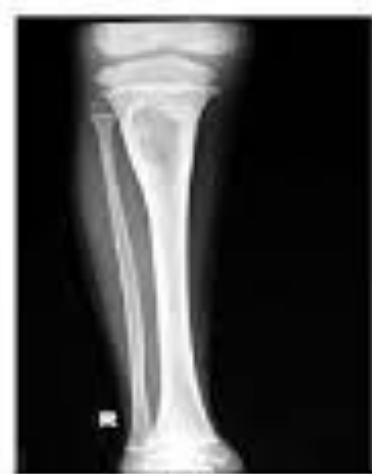

(c)
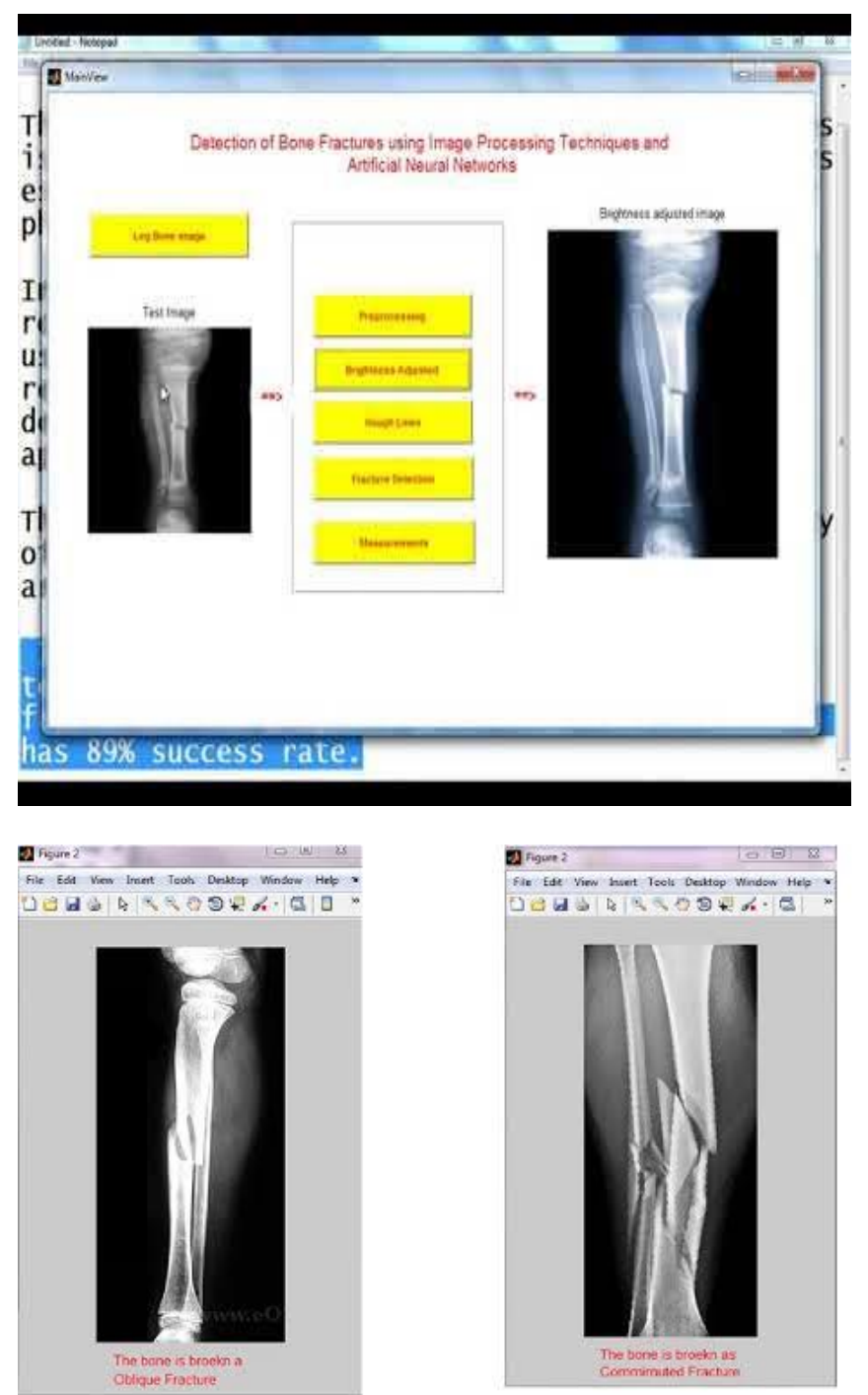

(2)

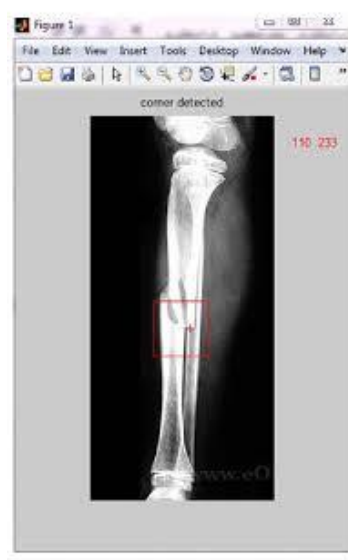

(b)

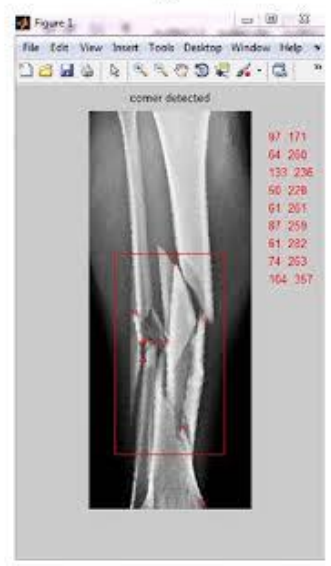

(b)

\section{COMPARISON OF RESULTS}

\begin{tabular}{|l|l|l|}
\hline Method & Accuracy & Precision \\
\hline ANN & $70 \%$ & $64 \%$ \\
\hline PNN & $75 \%$ & $68 \%$ \\
\hline BPNN & $82 \%$ & $76 \%$ \\
\hline
\end{tabular}

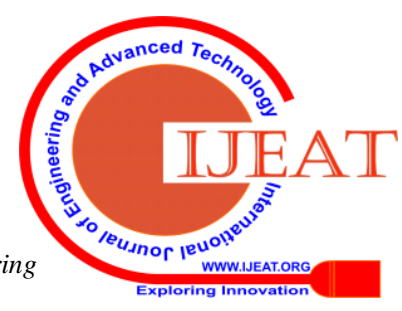




\section{Computer Based Bone Breakage Detection using Machine Learning Techniques}

\section{CONCLUSION}

Here we conclude our paper as the use of outfit characterization to break identification in $\mathrm{x}$-beam pictures was considered. Two sorts of highlights, in particular, surface highlights and shape highlights were separated from the x-beam pictures framing a sum of 12 highlights. Three parallel classifiers, SVM, BPNN and KNN were to fabricate gathering grouping models and during preparing, boosting strategy was utilized. The present investigation considers two-classifier and three-classifier gathering frameworks. The classifiers were assembled while changing the capabilities.

\section{REFERENCES}

1. Basha, C. M. A. K. Z., Sharon, K. O., Susmitha, K. L. S., \& Sai Sri, N. (2019). Advanced event attendance monitoring system. International Journal of Innovative Technology and Exploring Engineering, 9(1), 1930-1933.

2. Basha, C. Z., Srinivasa Rao, S., Lahari, P. L., Navya, B., \& Divya, S. V. S. (2019). An effective and robust computerized library management system. International Journal of Innovative Technology and Exploring Engineering, 9(1), 1647-1649.

3. Basha, C. Z., Simha, G. K. J., \& Krishna, Y. V. (2019). An efficient and robust fracture detection in femur bones. International Journal of Innovative Technology and Exploring Engineering, 9(1), 1954-1957.

4. Zeelan Basha, C., Vardhan, N. C. R., Gowtham, P., \& Tanuja, V. V. (2019). Automated student community portal. International Journal of Engineering and Advanced Technology, 9(1), 4353-4355.

5. Basha, C. Z., Krishna, A., \& Savarapu, P. R. (2019). Automatic detection of lung infection. International Journal of Recent Technology and Engineering, 8(3), 200-203.

\section{AUTHORS PROFILE}

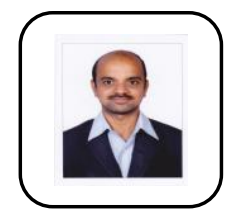

P.Neelakanteswara, is working as Assistant Professor in department of CSE in Koneru Lakshmaiah University. He is good at Image Processing.Interested in Coding .He Is an expert in data Science.He is having 9 years of experience.

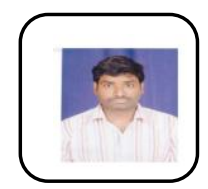

G.Kalyan Chakravarthy, is working as Assistant Professor in department of CSE in Koneru Lakshmaiah University. He is good at Image Processing.Interested in Coding .He Is an expert in data Science.He is having 11 years of experience.

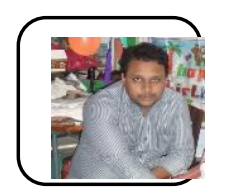

Ram Kumar Madupu, is working as Assistant Professor in department of CSE in Koneru Lakshmaiah University. $\mathrm{He}$ is good at Image Processing.Interested in Coding .He Is an expert in data Science.He is having 9 years of experience.

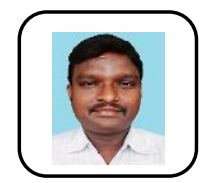

Dorababu Sudarsa, is working as Assistant Professor in department of CSE in Koneru Lakshmaiah University. He is good at Image Processing.Interested in Coding .He Is an expert in data Science.He is having 15 years of experience. 